\title{
CARTILAGE MATRIX REMODELLING DIFFERS BY DISEASE STATE AND JOINT TYPE
}

\author{
M-F. Hsueh ${ }^{1, *}$, V.B. Kraus ${ }^{1,2}$ and P. Önnerfjord ${ }^{3}$ \\ ${ }^{1}$ Duke Molecular Physiology Institute, Duke University School of Medicine, Duke University, \\ Durham, NC 27701, USA \\ ${ }^{2}$ Department of Medicine, Duke University School of Medicine, Duke University, Durham, NC 27701, USA \\ ${ }^{3}$ Department of Clinical Sciences Lund, Section of Rheumatology and Molecular Skeletal Biology, \\ Centre of Excellence in Biological and Medical Mass Spectrometry, Lund University, Lund, Sweden
}

\begin{abstract}
Dramatic alterations in mechanical properties have been documented for osteoarthritic (OA) cartilage. However, the matrix composition underlying these changes has not been mapped and their aetiology is not entirely understood. We hypothesised that an understanding of the cartilage matrix heterogeneity could provide insights into the origin of these OA-related alterations. We generated serial transverse cryo sections for 7 different cartilage conditions: 2 joint sites (knee and hip), 2 disease states (healthy and OA) and 3 tissue depths (superficial, middle and deep). By laser capture microscopy, we acquired 200 cartilage matrix specimens from territorial (T) and interterritorial (IT) regions for all 7 conditions. A standardised matrix area was collected for each condition for a total of $0.02 \pm 0.001 \mathrm{~mm}^{3}$ (corresponding to $20 \mu \mathrm{g}$ of tissue) from a total of 4800 specimens. Extracted proteins were analysed for abundance by targeted proteomics.

For most proteins, a lower IT/T ratio was observed for the OA disease state and knee joint type. A major cause of the altered IT/T ratios was the decreased protein abundance in IT regions. The collagenase-derived type III collagen neo-epitope, indicative of collagen proteolysis, was significantly more abundant in OA cartilage. In addition, it was enriched on average of 1.45 -fold in IT relative to T matrix.

These results were consistent with an elevated proteolysis in IT regions of OA cartilage, due to degenerative influences originating from synovial tissue and/or produced locally by chondrocytes. In addition, they offered direct evidence for dynamic remodelling of cartilage and provided a cogent biochemical template for understanding the alterations of matrix mechanical properties.
\end{abstract}

Keywords: Cartilage extracellular matrix, targeted proteomics, in situ trypsin digestion, laser capture microdissection, territorial matrix, interterritorial matrix.

*Address for correspondence: Ming-Feng Hsueh, PhD, Box 104775, Duke Molecular Physiology Institute, Duke University School of Medicine, Durham, NC 27701, USA.

Telephone: +19196812004Ｆax:+19196848907Ｅmail: mh167@duke.edu

\section{Introduction}

Articular cartilage is a highly-organised tissue with a low-friction surface that acts to facilitate joint mobility; its tightly-integrated structure withstands high mechanical load during movement (Bhosale and Richardson, 2008; Buckwalter and Mankin, 1998; Teeple et al., 2008). The articular cartilage above the tidemark can be separated into three distinct layers (superficial, middle and deep) differing from each other in histological characteristics, specifically, morphology and density of chondrocytes, collagen type, diameter, orientation and matrix protein composition (Buckwalter et al., 2005). The structural organisation of articular cartilage also differs regionally according to the distance of the matrix from chondrocytes (pericellular, territorial and interterritorial) (Bhosale and Richardson, 2008; Buckwalter and Mankin, 1998; Guilak et al., 2006). Moreover, until recently, it was not known that articular cartilage from different joint sites differ quite significantly in many protein constituents (Önnerfjord et al., 2012). These differences may have evolved in response to differences in the tissue mechanical environments or arisen secondarily, as a result of joint pathology.

Past studies, evaluating the differences in cartilage protein content at various depths, have focused on only one or two cartilage components (DiCesare et al., 1995; Lorenzo et al., 1998; Pfister et al., 2001) or used laser capture microdissection (LCM) to evaluate regional differences in gene expression (Fukui et al., 
2008a; Fukui et al., 2008b; Landis et al., 2003; Lui et al., 2015), but not protein. A comprehensive evaluation of the subregional differences of articular cartilage has not been performed and this, in part, may be due to the difficulties in distinctly separating subregions of cartilage tissue because of its stiffness and regional complexity. Dramatic alterations in mechanical properties have been documented for osteoarthritic (OA) cartilage (Wilusz et al., 2013). However, the protein matrix architecture underlying these changes has not been mapped and their aetiology is not entirely understood. We hypothesised that an understanding of the cartilage protein matrix architecture by joint site, depth and disease state could provide insights into the origin of these OA-related alterations. Moreover, an understanding of human cartilage protein heterogeneity across layers, subregions and joint type is important for elucidating the mechanisms of cartilage homeostasis and, in general, pathogenesis of OA cartilage. This information is also critical for recapitulation of native zonal architecture by tissueengineering approaches. Our major goal was to gain a comprehensive understanding of the protein network and composition of articular cartilage. Combining separation techniques, including transverse sectioning and LCM, we undertook a decisive phenotyping of cartilage heterogeneity by region, joint type and disease state, to gain an understanding of the location of sites with highest protein turnover.

\section{Material and Methods}

\section{Clinical cartilage specimen sources}

Under Duke Institutional Review Board approval, all articular cartilages were collected from Duke University Hospital as waste surgical specimens. Full thickness cartilage specimens from perilesional regions of the load-bearing area of hip and knee joints were obtained from patients with end-stage OA cartilage, who had arthroplasty surgery. Fullthickness healthy non-OA cartilage specimens were collected at the time of surgery for acute trauma; the absence of OA cartilage was determined by the surgeon and confirmed by macroscopic inspection upon acquisition of the sample in the laboratory. All specimens were snap-frozen, by directly inserting tubes into dry ice, and stored at $-80{ }^{\circ} \mathrm{C}$.

\section{Cartilage dissection and laser capture microdissection}

Sample preparation and workflow are illustrated in Fig. 1a. Cartilage specimens were embedded in Tissue-Tek ${ }^{\circledR}$ O.C.T. (Sakura, Alphen aan den Rijn, The Netherlands) for cryosectioning. The entire cartilage was sectioned transversely (12 $\mu$ m-thick sections) starting from the cartilage surface and progressing to the deep zone. For LCM, tissue sections were mounted onto slides covered with polyethylene naphthalate (PEN)-membrane (ZEISS, Oberkochen,
Germany). 20 adjacent sections were collected at different distances from the surface to represent the superficial $(0-240 \mu \mathrm{m})$, middle $(480-720 \mu \mathrm{m})$ and deep (960-1200 $\mu \mathrm{m})$ layers. Twenty sections between each layer were skipped to avoid cross-contamination. Decellularisation was performed, as previously described, to remove chondrocyte intracellular proteins from the cartilage extracellular matrix proteome (Hsueh et al., 2016). This step resulted also in the removal of the aqueous embedding media that can adversely affect the liquid chromatography step. Fixation was performed by dropping for $5 \mathrm{~s}$ ice-cold $70 \%$ ethanol onto the frozen sections. For LCM, we obtained approximately 200 lasermicrodissected specimens from 10 sections for each distinct subregion (interterritorial and territorial matrix) at each cartilage depth (superficial, middle and deep) and derived from healthy and OA knees and hips. A total of 24 groups of sections, including 3 depths, 2 types of joints, 2 types of disease status and 2 types of subregions, were derived from the following subjects: a 56 year-old female patient, who had knee arthroplasty surgery, a 64 year-old female patient, who had hip arthroplasty surgery with end-stage OA cartilage, a 48 year-old female patient, who had acute knee trauma surgery, and a 35 year-old male patient, who had acute hip trauma surgery. In total, 4800 specimens were acquired by microdissection, representing each joint site, region, depth and disease state. To distinguish the subregions of cartilage to be dissected by laser microdissection, tissue sections were stained for $1 \mathrm{~min}$ with toluidine blue dye. Sections were dehydrated through exposure to increasing concentrations of ethanol (70\%, $95 \%$ and $100 \%)$. Laser capture microdissection, using a ZEISS PALM microbeam system (ZEISS), provided the means to collect cartilage matrix from the territorial and interterritorial regions. These regions were distinguished by histological staining characteristics (Henrikson, 1997), i.e. an abrupt decline in toluidine blue staining demarcated the shift from territorial to interterritorial regions. The abrupt transition from dark to light toluidine blue occurred on average $27.4 \pm 9.6 \mu \mathrm{m}$ from the cell void; this landmark was distinct in both healthy and osteoarthritic cartilage. The captured subregions of matrix were collected by wet collection method. In brief, ammonium bicarbonate (AmBic, SigmaAldrich, St. Louis, MO, USA) buffer was added to the cap of a $600 \mu \mathrm{L}$ microfuge tube that was held in place above the sections. The subregion samples were catapulted from the slide and captured by the wet inner surface of the cap. The same approximate volume of extracellular matrix $\left(0.02 \pm 0.001 \mathrm{~mm}^{3}\right.$ yielding approximately $20 \mu \mathrm{g}$ tissue) was collected for each specimen.

\section{Specimen preparation for mass spectrometry analysis}

By a quick spin of the microfuge tubes, groups of 200 LCM-harvested specimens for a particular region were 
pooled by immersion in $50 \mathrm{mM}$ AmBic, containing $0.2 \%$ RapiGest (Waters Corporation, Milford, MA, USA), $0.1 \mathrm{U}$ of streptococcal hyaluronidase (Seikagaku, Tokyo, Japan), 100 mM 6-aminocaproic acid, $1 \mathrm{mM}$ benzamidine, $1 \mathrm{mM}$ EDTA and $5 \mathrm{mM}$ N-ethylmaleimide $\mathrm{pH} 7$, and heated for $3 \mathrm{~h}$ at $37^{\circ} \mathrm{C}$. The specimens were further processed by reduction with $4 \mathrm{mMDTT}$ for $30 \mathrm{~min}$ at $56^{\circ} \mathrm{C}$ and alkylation with $16 \mathrm{mM}$ iodoacetamide for $1 \mathrm{~h}$ at room temperature in the dark. Trypsin digestion was performed with $2 \mu \mathrm{g}$ of Trypsin Gold (Promega, Madison, WI, USA) for $16 \mathrm{~h}$ at $37^{\circ} \mathrm{C}$. Subsequently, the tryptic peptides were diluted with $0.5 \mathrm{M}$ AmBic and filtered through a $30 \mathrm{kDa}$ filter (Pall Life Sciences, Port Washington, NY, USA) followed by a reverse-phase C18 column (The Nest group, Southborough, MA, USA) to remove peptides containing polysaccharide chains and residual salt.

\section{Targeted mass spectrometry analysis}

Targeted data acquisition using multiple reaction monitoring (MRM) was performed as previously described (Hsueh et al., 2016; Müller et al., 2014). In brief, processed sample aliquots were quantified using a TSQ Vantage triple quadrupole mass spectrometer (Thermo Scientific, Waltham, MA, USA). The mass spectrometer was operated with both Q1 and Q3 settings at $0.7 \mathrm{Da}$ resolution. The on-line reversephase chromatographic separation was performed on an Easy nano-LC system (Thermo Scientific) using a linear binary gradient. A standard mixture of tryptic peptides was run between the samples to check the system performance. The monitored peptide sequence, transitions and collision energies for the MRM were as previously described (Müller et al., 2014). In the present study, we monitored a total of 45 proteins and obtained reliable (matching transitions and retention times) and detectable signals from 30 proteins.

\section{Data analysis}

MRM data were analysed using the Skyline 2.0 software (MacCoss Lab Software, University of Washington, MD, USA). The peak area of MS2 fragment ions (MS/MS), within the expected retention time of the peak, ensured the identity of the peak, as measured by synthetic peptides during optimisation; MS2 fragment ions for each peptide were summed for 3-5 transitions for MRM experiments. When more than one peptide was available for a particular protein, the peak area of one peptide across the specimens was standardised and the mean standardised values of the peptides derived from one protein were used to represent the protein abundance ( $Z$ score). We calculated a ratio to quantify the relative abundance of protein in the interterritorial/territorial (IT/T) matrices; values were log-transformed to achieve normality. For MRM, we compared the protein abundance across the specimens by relative quantification. Multivariable regression was performed with multiple independent factors, joint site (hip/knee), disease state (healthy/ OA), depth (superficial, middle, deep), subregions (territorial/interterritorial) and age of the cartilage, to evaluate their association with the continuous outcome response variable (protein abundance). The model evaluated the predicted response for each specific factor after controlling for the other four factors. Then, we conducted the Holm step-down procedure to examine the outcomes controlling the family-wise error rate. The $p$ values from the multivariable regression of all monitored proteins were sorted from low to high and then the lowest $p$ value was tested with a Bonferroni correction involving all tests, the second test was tested with a Bonferroni correction involving one less test and so on for the remaining tests (Abdi, 2010). Statistical significance for each factor and the overall model was reported at $95 \%$ confidence level $(p<0.05)$. The multivariable analyses were performed using $\mathrm{JMP}^{\circledR}$ Pro 11.2 (SAS, Cary, NC, USA). All the graphs were prepared in Microsoft Excel, PowerPoint 2013 or GraphPad Prism 5.

\section{Results}

\section{Protein abundance distribution patterns in cartilage}

Articular cartilage subregions were isolated using LCM. A representative cartilage section is shown in Fig. 1b (left panel) along with microdissected samples from the territorial matrix (middle panel) and interterritorial matrix (right panel). The transition from territorial to interterritorial matrix was demarcated by an abrupt change in the intensity of toluidine blue staining from dark to light, which reflects the difference in sulphated glycosaminoglycans (Henrikson, 1997). Therefore, the size of the territorial matrix could be estimated by the radial distance of this change in staining intensity from the chondrocyte surface. The average radial extent (outer margin) of the territorial matrix from the cell surface was $26.1 \pm 6.2 \mu \mathrm{m}$ in healthy knee cartilage and it was significantly less in OA knee cartilage $(19.1 \pm 8.8 \mu \mathrm{m})$. This landmark was also observed in hip cartilage: the average radial extent of the territorial matrix from the cell surface was $34.4 \pm 10.6$ and $26.2 \pm 5.1 \mu \mathrm{m}$ in healthy and OA hip cartilage, respectively and again was significantly less in OA cartilage. Overall, the radial extent (on average $30.6 \pm 9.4 \mu \mathrm{m}$ ) observed in hip cartilage was greater than the one in knee cartilage (on average $23.3 \pm 8.1 \mu \mathrm{m})$.

\section{Protein abundance by joint site}

In contrast to all previous studies (Hsueh et al., 2016; Müller et al., 2014; Önnerfjord et al., 2012), in the current study, the proteomic results represented protein abundance in uniformly-sized cartilage samples (Table 1). Among the 30 proteins we monitored, joint site differences were observed in 19 proteins after 
controlling for other factors; 12 of them remained significant, based on the Holm step-down procedure (Table 1). These proteins included aggrecan core protein (G1 and G2 domains) and cartilage oligomeric matrix protein (COMP), both significantly enriched in hip cartilage (Fig. 2a). Other proteins enriched in hip cartilage included chondroadherin (CHAD), cartilage intermediate layer proteins 1-2, 2-1 and 2-2 (CILP12, CILP 2-1, CILP 2-2), type XI collagen (COBA2), versican core protein (CSPG2), dermatopontin (DERM), fibromodulin (FMOD), hyaluronan and proteoglycan link protein 1 (HPLN1), serine protease HTRA1 (HTRA1), matrilin-3 (MATN3), matrix Gla protein (MGP), biglycan (PGS1) and thrombospondin-1 and -4 (TSP1 and TSP4) (Table 1). In contrast to these proteins, one protein, mimecan (MIME), was significantly enriched in knee cartilage (Fig. 2a).

\section{Protein abundance by depth}

Based on results of the multivariable analyses, most of the proteins were enriched in the deep layer of cartilage, including CILP2-1, fibrillin-1 (FBN1), MGP, aggrecan core protein subdomain (PGCA-3), PGS1, decorin (PGS2), prolargin (PRELP), Target of Nesh$\mathrm{SH} 3$ protein (TARSH) and TSP1 (Fig. 2b). Changes in

a)

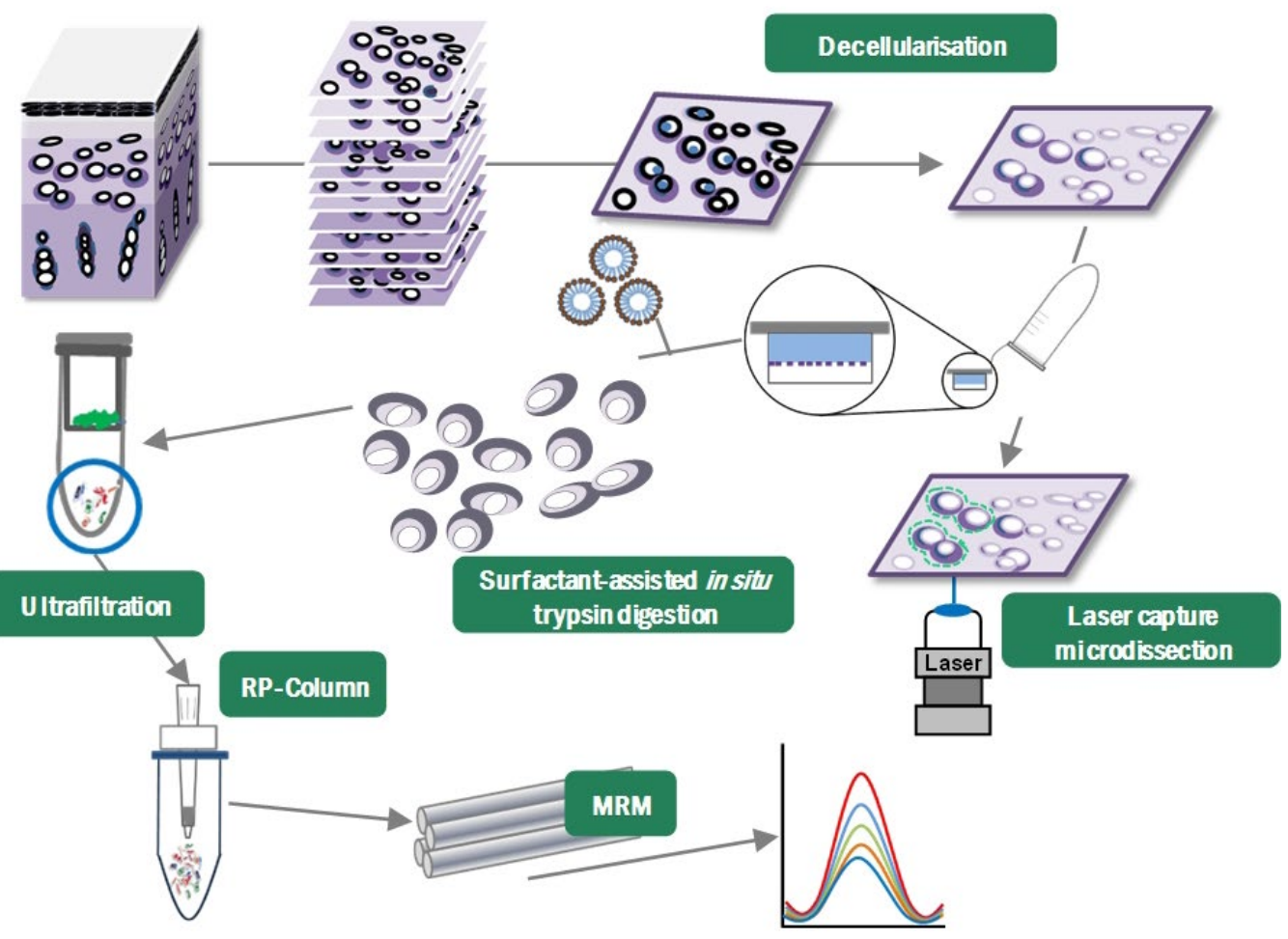

b)
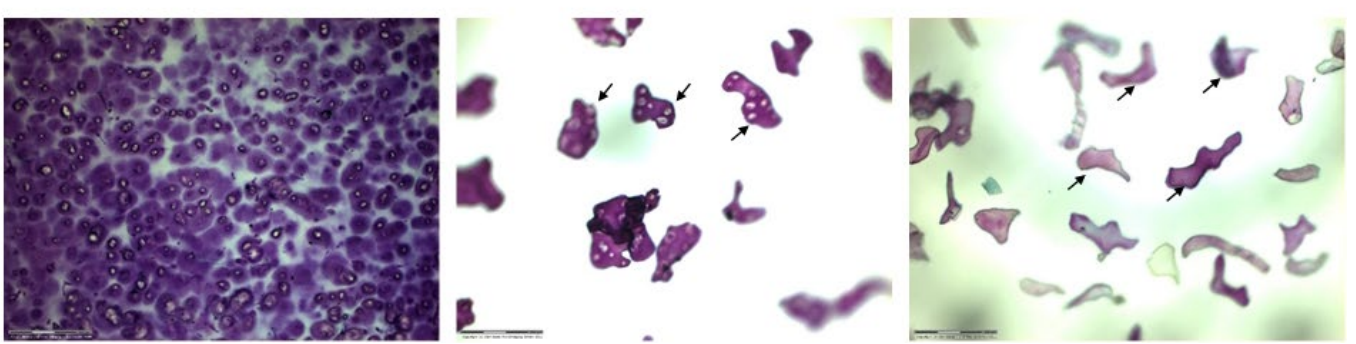

Fig. 1. Workflow of laser capture microdissected cartilage tissue proteomic analysis. (a) Sections were generated parallel to the articular cartilage surface. Chondrocytes were removed according to Hsueh et al. (2016). Desired subregions were collected separately by laser capture microscopy (LCM). Microdissected samples were collected with AmBic buffer in the caps of microfuge tubes. LCMharvested sections were treated by in situ trypsin digestion method with surfactant, as previously described (Hsueh et al., 2016). Extracts were ultrafiltrated to remove interfering GAGs and the residual salt was removed by reverse-phase (RP) spin column. Proteomic analyses were performed by LCtriple quadrupole MS (quantitative MRM proteomics). (b) The transition from dark to light toluidine blue dye staining marked the regions designated as territorial vs. interterritorial, respectively (left). Microscopic examination of laser captured territorial (middle panel) and interterritorial (right panel) matrix (in the cap of collection tube). 
Table 1. Proteins significantly enriched. The results were generated by multivariable regression analysis using four independent factors evaluated simutaneously: age, joint site, disease state, cartilage depth and subregion of cartilage. Text in bold highlights proteins with a $p$ value lower than 0.05 , indicating that, after controling for the other variables, there was a significant independent association of the protein with either joint site, disease state, depth or subregion. Text with an underlined highlights the proteins with a $p$ value lower than 0.05 after the Holm step-down procedure.

* Only one peptide was available for a particular protein. \# Mean value was higher in the knee joint.

$\sim$ Mean value was higher in OA joints. ${ }^{\wedge}$ Mean value was higher in the superficial layer.

\begin{tabular}{|c|c|c|c|c|c|c|}
\hline \multirow[b]{2}{*}{ Accession } & \multirow[b]{2}{*}{ ID } & \multirow[b]{2}{*}{ Protein } & \multicolumn{4}{|c|}{$p$ value } \\
\hline & & & Joint & Disease & Depth & Subregion \\
\hline 015335 & CHAD & Chondroadherin & $\leq .0001$ & $\leq .0001^{\sim}$ & 0.4351 & 0.5551 \\
\hline O75339 & CILP1-1 & Cartilage intermediate layer protein $1-1$ & 0.0747 & 0.3249 & 0.0737 & 0.2967 \\
\hline O75339 & CILP1-2 & Cartilage intermediate layer protein 1 -2 & $\leq .0001$ & 0.0129 & $\underline{0.0004}$ & 0.0295 \\
\hline Q8IUL8 & CILP2-1 & Cartilage intermediate layer protein 2-1 & $\underline{0.0003}$ & 0.1404 & 0.0304 & 0.3735 \\
\hline Q8IUL8 & CILP2-2 & Cartilage intermediate layer protein 2-2 & 0.0141 & 0.2798 & 0.2799 & 0.0728 \\
\hline P02461 & CO3A1 & Collagen alpha-1(III) chain & 0.2047 & 0.8223 & 0.7824 & 0.9244 \\
\hline P12111 & CO6A3 & Collagen alpha-3(VI) chain & 0.2562 & 0.8125 & 0.2558 & 0.3266 \\
\hline P20849 & CO9A1 & Collagen alpha-1(IX) chain * & 0.1493 & 0.7349 & 0.0682 & 0.9254 \\
\hline P13942 & СОВА2 & Collagen alpha-2(XI) chain * & $\leq .0001$ & $0.0037^{\sim}$ & 0.0545 & 0.2038 \\
\hline P49747 & COMP & Cartilage oligomeric matrix protein & $\underline{0.0002}$ & $\underline{0.0007}$ & $\leq .0001$ & 0.0643 \\
\hline P13611 & CSPG2 & Versican core protein & 0.0148 & 0.6137 & 0.2340 & 0.0876 \\
\hline Q07507 & DERM & Dermatopontin * & 0.0223 & 0.5285 & $\underline{0.0015}$ & 0.2195 \\
\hline P35555 & FBN1 & Fibrillin-1 & 0.6710 & 0.8515 & 0.0366 & 0.1283 \\
\hline P02751 & FINC & Fibronectin & 0.8329 & 0.6314 & $\underline{0.0006}$ & 0.3343 \\
\hline Q06828 & FMOD & Fibromodulin & 0.0150 & 0.1035 & 0.0657 & 0.0564 \\
\hline P10915 & HPLN1 & $\begin{array}{l}\text { Hyaluronan and proteoglycan link } \\
\text { protein } 1 \\
\end{array}$ & $\leq .0001$ & 0.5130 & 0.0822 & 0.0055 \\
\hline Q92743 & HTRA1 & Serine protease HTRA1 & $\leq .0001$ & 0.0332 & $\underline{0.0018}$ & 0.0681 \\
\hline P51884 & LUM & Lumican * & 0.4495 & 0.4584 & 0.3424 & 0.1053 \\
\hline 015232 & MATN3 & Matrilin-3 & $\underline{0.0009}$ & 0.7281 & 0.4160 & 0.5444 \\
\hline P08493 & MGP & Matrix Gla protein & $\leq .0001$ & $\leq .0001$ & 0.0049 & 0.0196 \\
\hline P20774 & MIME & Mimecan & $0.0052^{\#}$ & 0.4642 & $0.0283^{\wedge}$ & 0.3892 \\
\hline P98160 & PGBM & Perlecan & 0.0703 & 0.3945 & 0.1661 & 0.7221 \\
\hline P16112 & PGCA-1/2 & Aggrecan core protein G1 \& G2 & $\leq .0001$ & 0.0029 & $\underline{0.0010}$ & 0.0300 \\
\hline P16112 & PGCA-3 & Aggrecan core protein G3 & 0.0807 & 0.4298 & 0.0028 & 0.7314 \\
\hline P21810 & PGS1 & Biglycan & 0.0075 & 0.7316 & 0.0040 & 0.3318 \\
\hline P07585 & PGS2 & Decorin & 0.0840 & 0.0360 & 0.0326 & 0.0377 \\
\hline P51888 & PRELP & Prolargin * & 0.1475 & 0.0941 & 0.0025 & 0.3946 \\
\hline Q7Z7G0 & TARSH & Target of Nesh-SH3 * & 0.2773 & 0.0207 & 0.0120 & 0.2719 \\
\hline P07996 & TSP1 & Thrombospondin-1 & $\underline{0.0007}$ & 0.0283 & 0.0378 & 0.1355 \\
\hline P35443 & TSP4 & Thrombospondin-4 & $\underline{0.0017}$ & 0.4409 & 0.3326 & 0.2062 \\
\hline P02461 & $\begin{array}{c}\text { COL3- } \\
\text { neo }\end{array}$ & Collagen type III neo-epitope & $\underline{0.0003}^{\sharp}$ & $\leq .0001$ & 0.0139 & 0.5999 \\
\hline
\end{tabular}


a) Protein abundance by joint site
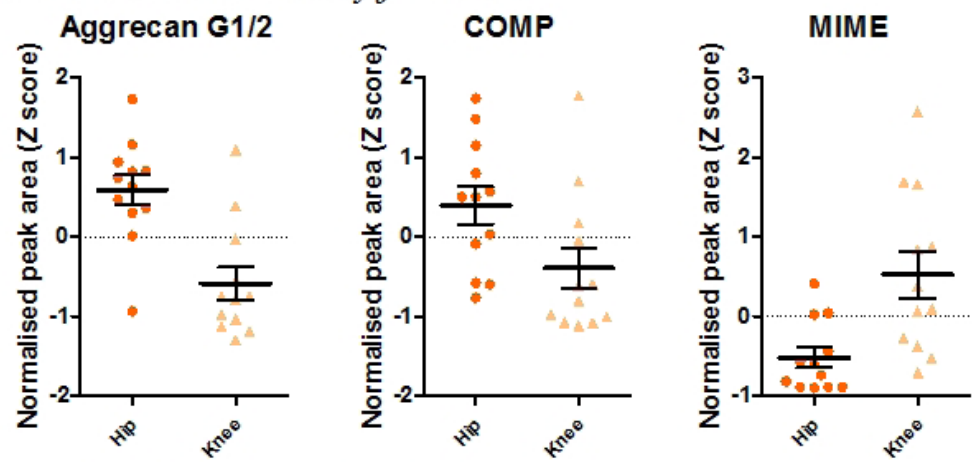

b) Protein abundance by depth
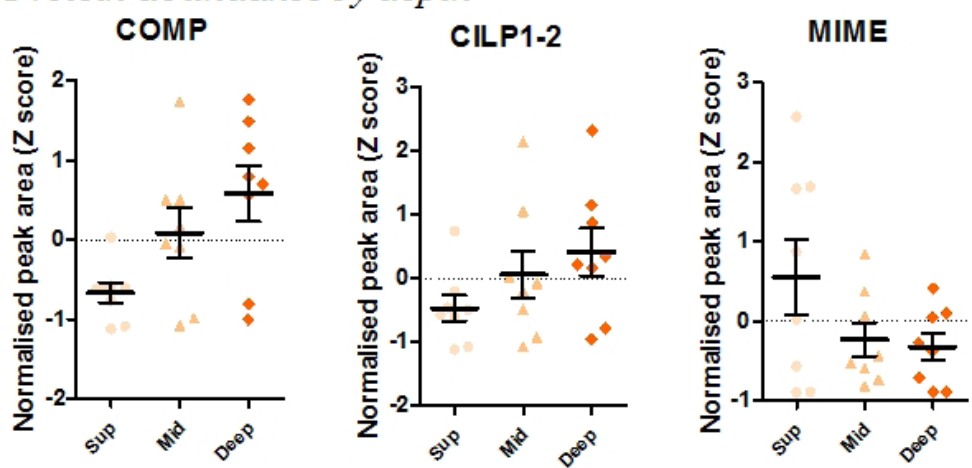

c) Protein abundance by disease state
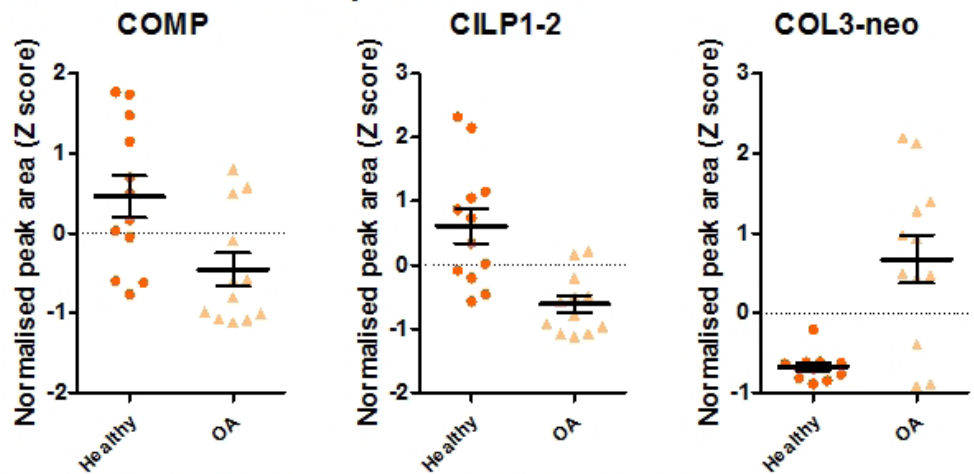

d) Protein abundance by cartilage subregion
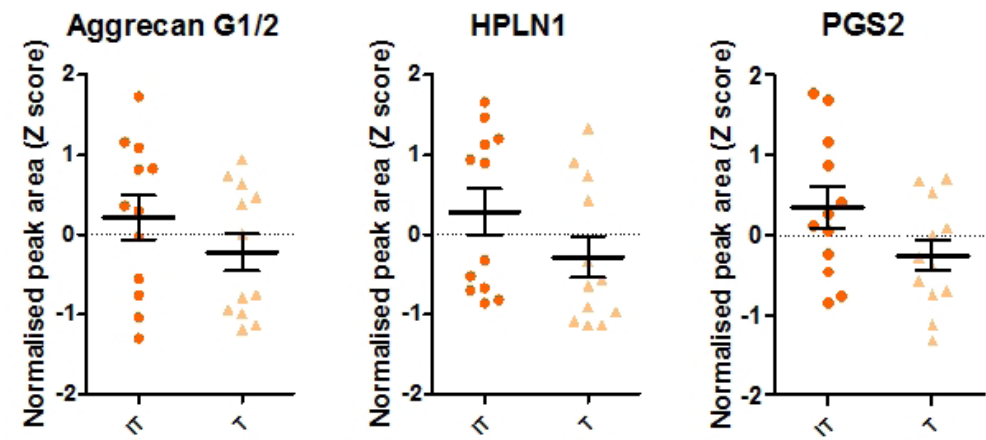

Fig. 2. Cartilage matrix protein abundance distribution patterns. Representative proteins were categorised by (a) joint type, (b) depth of cartilage, (c) disease state and (d) subregion [interterritorial (IT) vs. territorial (T)].
CILP1-2, COMP, DERM, fibronectin (FINC), HTRA1 and aggrecan core protein subdomain (PGCA-1/2) were significant, based on the Holm step-down procedure. In contrast to these proteins, MIME was significantly enriched in the superficial layer (Fig. 2b).

Protein abundance by disease state

We also investigated cartilage protein composition by disease state. CILP1-2, HTRA1, PGCA-1/2,
PGS2, TARSH and TSP1 were all significantly less abundant within OA compared to healthy cartilage tissue (Fig. 2c). COMP remained significantly less abundant within OA cartilage after the Holm stepdown procedure. CHAD, MGP and COBA2 were significantly more abundant in $\mathrm{OA}$ cartilage tissue; changes in CHAD and MGP were significant, based on the Holm step-down procedure (Table 1). 


\section{Proteolytic neoepitope}

The collagenase-derived type III collagen neo-epitope [COL3-neo, IAGITGAR (949-956)], indicative of proteolytic degradation of cartilage, was significantly more abundant in OA cartilage tissue [healthy - $0.67 \pm 0.16$; OA $0.67 \pm 1.03$ (Z score)] (Fig. 2c) and was enriched, on average, of 1.45 -fold in IT relative to T matrix. COL3-neo was also significantly enriched in knee compared to hip cartilage (knee $0.35 \pm 1.20$; hip - $0.35 \pm 0.63)$; it achieved significance for joint type and disease state based on the Holm step-down procedure. COL3-neo was also significantly enriched in deep layers of cartilage (superficial $-0.37 \pm 0.72$; middle $0.11 \pm 1.13$; deep $0.26 \pm 1.11$ ) (Table 1 ).

\section{Protein abundance by cartilage subregion}

Unique to this study, we directly investigated protein abundance by cartilage subregion (territorial and interterritorial regions). CILP1-2, HPLN1, MGP, PGCA-1/2 and PGS2 were significantly more abundant in the interterritorial compared to the territorial region (Fig. 2d).

\section{Relative protein abundance of interterritorial/} territorial regions is primarily related to disease state

To evaluate protein alterations in the horizontal plane from the chondrocyte surface, we evaluated the relative abundance of matrix proteins as ratio of interterritorial versus territorial regions (IT/T ratio). The abundance of the aggrecan G1/G2 and G3 domains was illustrative of major differences in IT/T ratios. The G1/G2 domain was enriched in the interterritorial matrix of healthy cartilage, but was approximately equally abundant within interterritorial and territorial subregions of OA cartilage (Fig. 3a). The pattern of abundance of the aggrecan G3 domain was the opposite: G3 was enriched in the territorial matrix of healthy cartilage rather then in the interterritorial matrix of $\mathrm{OA}$ cartilage (Fig. 3b). Interestingly, the IT/T ratio of the aggrecan core protein did not differ by joint site (hip versus knee) or cartilage depth.

Multivariable regression analyses were used to take all biological factors simultaneously into consideration to identify the independent or specific associations of the biological factors with the protein alterations (Table 2). Generally, disease state and joint type were the predominant factors affecting the IT/T ratio for most proteins. The IT/T ratios of only 3 proteins were independently affected by cartilage depth [type IX collagen (CO9A1), FBN1 and PGS1]. A reduced IT/T ratio in OA cartilage, as seen for HTRA1 and CILP2-2 (Table 2), might be due to a reduction in protein abundance in the interterritorial matrix, an increased abundance in the territorial matrix or a combination of both factors. Direct quantification of protein abundance within these subregions revealed that a decreased protein abundance in interterritorial regions was the major cause of the altered IT/T ratios in OA cartilage (Fig. 4a).

For some proteins, the IT/T ratio was also affected by joint type (Table 2). For instance, type VI collagen (CO6A3), CO9A1, FBN1, HTRA1, MGP, perlecan (PGBM), PGCA-3 and PGS1 were relatively enriched in the interterritorial compared to the territorial

a)

Aggrecan G1/2 Disease state

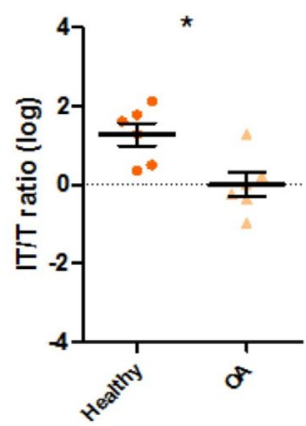

b)

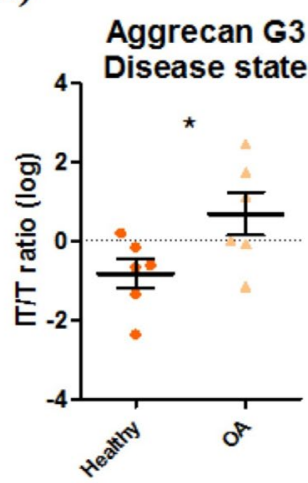

Joint

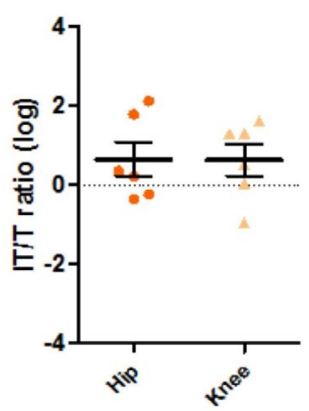

Joint

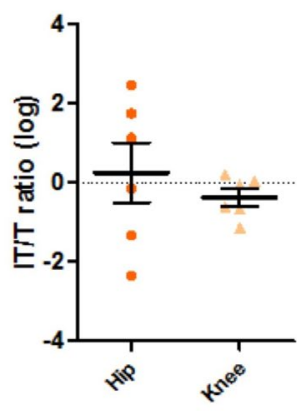

Depth

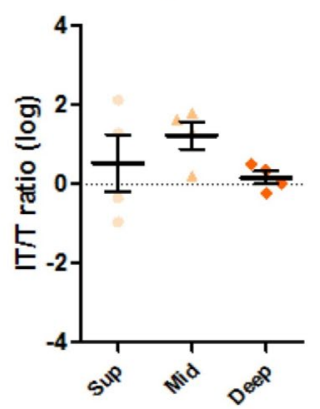

Depth

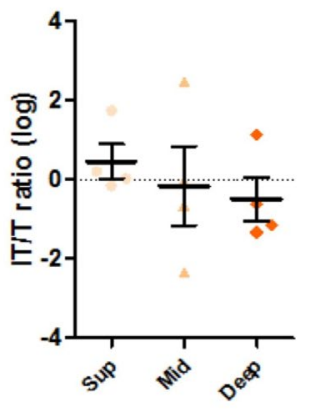

Fig.3. Interterritorial (IT)/territorial (T) matrix ratio of cartilage matrix proteins. The IT/T ratios of (a) aggrecan G1/G2 and (b) G3 proteins categorised by disease state, joint type and depth of cartilage. The IT/T ratio of aggrecan G1/G2 was significantly higher in healthy cartilage; in a reverse pattern, the ratio of aggrecan G3 was significantly lower in healthy cartilage $(p<0.05$, measured by $t$ test). The IT/T ratio of the aggrecan core protein did not differ by joint site (hip vs. knee) or cartilage depth. Sup: superficial layer. Mid: middle layer. Deep: deep layer. 


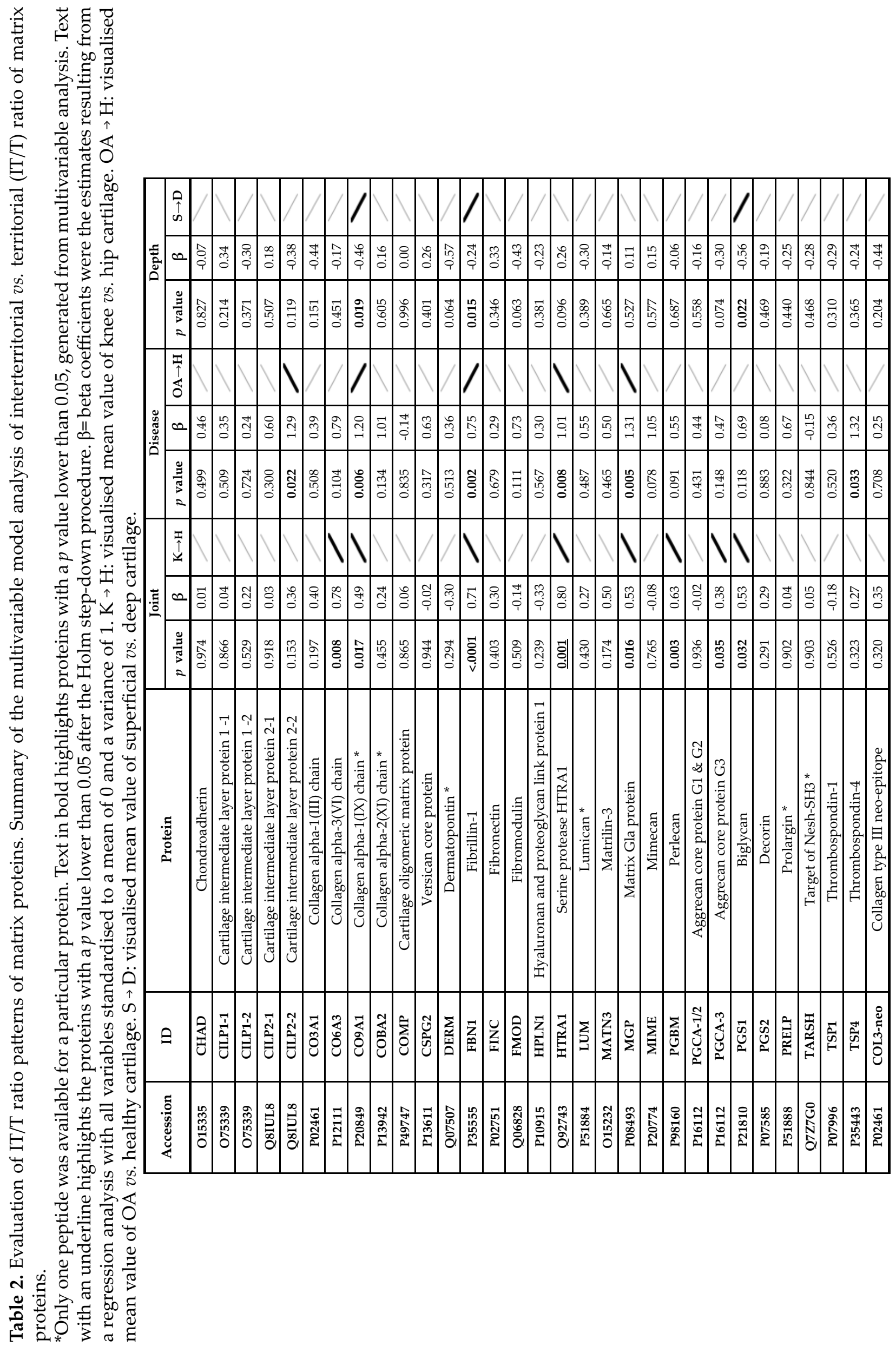


a)

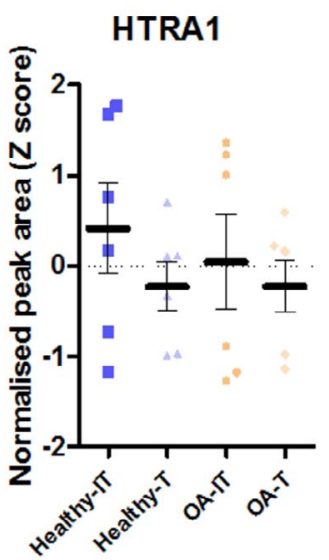

b)

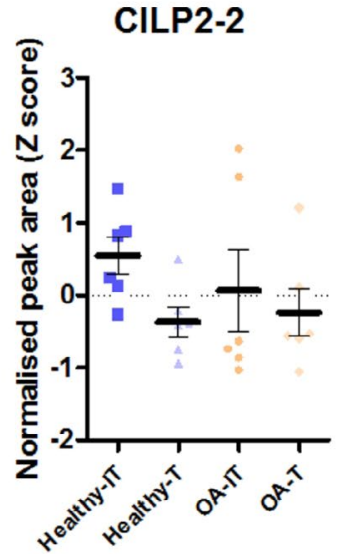

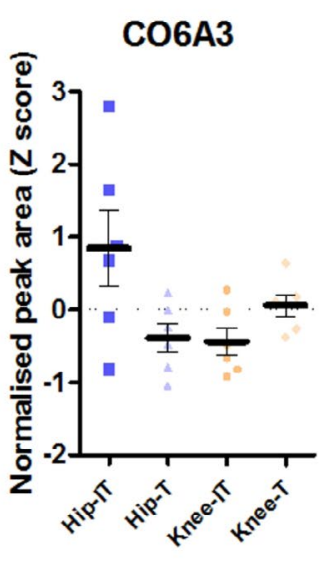

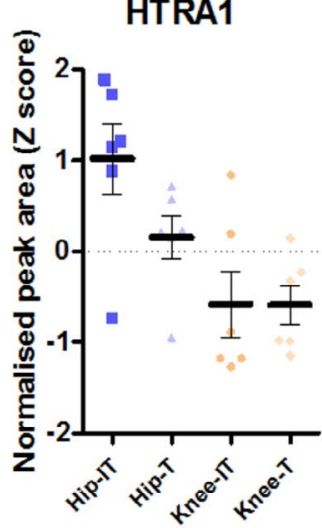

Fig. 4. Abundance patterns of proteins, with significant IT/T ratio differences, by disease state and joint type. Protein abundance distribution categorised by (a) disease state and (b) joint type. The abundance of CILP2-2 and HTRA1 proteins within the IT subregion was consistent with a dramatic protein degradation or loss in OA cartilage. CO6A3 was enriched in the hip interterritorial domain with a different pattern compare to the knee. HTRA1 was also enriched within the hip interterritorial region.

matrix of hip cartilage compared to the interterritorial matrix of knee cartilage (Table 2). Among these proteins, only HTRA1 remained significant based on the Holm step-down procedure. Direct quantification of protein abundance revealed that both, CO6A3 and HTRA1, were enriched in hip interterritorial matrix (Fig. 4b).

\section{Discussion}

Our newly developed methodology to comprehensively map protein abundance within articular cartilage tissue yielded some novel insights related to cartilage and alterations in osteoarthritis. We used LCM, the only tool for separating the cartilage territorial and interterritorial matrix. To our knowledge, no prior study has investigated protein variations in cartilage subregion using LCM. Since extracellular proteins of both, the territorial and interterritorial regions, are generated by chondrocytes, it is impossible to identify the bona fide differences in these subregions only based on genomic (gene expression) strategies - that, in the past, have been relied upon to identify zonal differences (Fukui et al., 2008b; Grogan et al., 2013). We calculated the ratio of protein abundance in the interterritorial relative to the territorial region to evaluate the alteration in protein abundance between these two subregions with respect to joint type, disease state and cartilage depth. Importantly, the ratio was determined from tissue specimens of equal volumes originating from the same horizontal plane, thereby providing a mean of direct comparison. Interestingly, the alteration of this ratio was primarily related to disease state and joint type. Namely, for most proteins, a lower absolute protein abundance in the interterritorial regions of $\mathrm{OA}$ and hip cartilages was responsible for the disease and joint site-related changes of the $\mathrm{IT} / \mathrm{T}$ ratio. In addition, based on toluidine staining, we observed an overall diminution in the size of the territorial matrix (defined by intense staining) in $\mathrm{OA}$ cartilage and an accompanying increase in the size of the interterritorial matrix (defined by less intense staining and reflecting proteoglycan depletion). These alterations were clear evidence of matrix protein reorganisation due to disease.

Type III collagen molecules with unprocessed $\mathrm{N}$-pro-peptides are present in the extracellular matrix of adult human and bovine articular cartilages as polymers covalently crosslinked to type II collagen (Wu et al., 2010). By immunohistochemical staining with a monoclonal antibody specific for a conformational epitope of the globular N-propeptide domain (Eyre et al., 2006), it was shown that type III collagen is predominantly concentrated in the territorial matrix surrounding individual chondrocytes and chondrocyte clusters, strongly implying biosynthesis and deposition by the chondrocytes themselves (Hosseininia et al., 2016). The degradation neo-epitope of collagen type III was first discovered in cartilage explants and their culture media upon treatment with injurious compression and exogenous cytokines (Wang et al., 2016). This study confirmed that this collagen type III neoepitope was generated in vivo in OA cartilage tissue. The cleavage site of the neo-epitope is in a region containing repeats of the motif GXP. The cleavage occurs at the Gly-Ile bond, which is analogous to the $3 / 4-1 / 4$ cleavage site of collagen type II (Howes et al., 2014; Wang et al., 2016). According to the MEROPS database (Rawlings et al., 2010) and consistent with the literature (Billinghurst et al., 1997; Rawlings et al., 2010; Zhen et al., 2008), this epitope is generated by metallopeptidases (MMP-1,-8,-12 and -13) of which, MMP-1 and MMP-13 were both identified in these 
specimens (Hsueh et al., 2016). Therefore, the presence of this peptide indicates regions of high proteolytic activity. The higher content of collagenase-derived collagen type III neo-epitope (COL3-neo), observed in OA interterritorial regions, could contribute to a loss of extracellular matrix (ECM) mechanical properties in OA cartilage. Our data were remarkably consistent with previous studies that have spatially mapped the in situ biomechanical properties of articular cartilage (normal human, porcine and murine) by atomic force microscopy. In healthy cartilage, the elastic modulus of the pericellular matrix (PCM) is significantly smaller than the one of the ECM (Darling et al., 2010). However, in early OA human articular cartilage, the elastic modulus of both the PCM and ECM was reduced by $30 \%$ and $45 \%$, respectively (Wilusz et al., 2013). These results are consistent with our observations that the protein abundance varied in the horizontal plane from the chondrocyte surface, with greater declines further from the chondrocyte surface. Therefore, our results provided potential insights related to the specific protein alterations responsible for the change in mechanical properties in OA cartilage.

Mechanical overload can lead to maladaptive cellular responses and cartilage degradation, in part mediated by mechanosensitive ion channels, such as PIEZO 1 and 2 (Lee et al., 2014). In addition, in conjunction with proinflammatory mediators, originating from chondrocytes or the milieu of the joint, such as synoviocytes, mechanical overload can activate signalling pathways that release chondrocytes from growth arrest and lead to the production of inflammation-related genes, including nitric oxide synthase-2, cyclooxygenase-2 and cartilage proteases, such as MMPs-1, 3 and 13 and disintegrin and metalloproteinase with thrombospondin motifs (ADAMTS) -4 and -5 (Goldring et al., 2011). Intriguingly, the altered load on osteoblasts and osteocytes also leads to metalloproteinase production (MMPs-3 and -13) due to chondrocytes production mediated by bone production of 14-3-3 epsilon (Priam et al., 2013). Increased matrix-degrading proteinases generate protein fragments, which can promote further inflammation and cartilage catabolism contributing to the onset or progression of osteoarthritis (Goldring, 2012). The collagenase-derived collagen type III neo-epitope, indicative of collagen proteolysis, was significantly more abundant in OA cartilage and tended to be enriched in the interterritorial matrix. Moreover, this neo-epitope was enriched in the deep layer of cartilage tissue. According to our previous work, metallopeptidases capable of generating this neo-epitope (MMP-1) tended to be enriched in the deep layer, whereas metalloproteinase inhibitors (TIMP1 and 2) tended to be enriched in the superficial layer (Hsueh et al., 2016). Our results suggested that excessive matrix catabolism dominated within the interterritorial and deep regions and was consistent with a dominant proteolytic influence originating from local aberrantly-loaded chondrocytes and/or bone cells. For instance, matrix catabolism originating from proteases in the synovial fluid or chondrocytes may have a predominant effect on the interterritorial regions to generate more neo-epitope. Alternatively, the collagen degradation neo-epitope may be preferentially cleared in the territorial region by cell-associated mechanisms. Increased degradation in OA cartilage, combined with insufficient protein synthesis, particularly in the interterritorial region, would, as we observed, increase the IT/T ratio of collagen type III neo-epitope and reduce the IT/T ratio of the majority of the other matrix components as an end-result of matrix proteolysis.

Among the unexpected and noteworthy findings, we observed that collagen type VI (CO6A3) was present in the interterritorial domain of both healthy hip and knee cartilage and equally abundant or even slightly enriched in deep layers. These results are in striking contrast to the data in the literature, generated by antibody-based immunohistochemistry, showing that collagen VI is exclusively localised into the pericellular and territorial matrix (Guilak et al., 2006; Soder et al., 2002). Of note, these prior immunohistochemistry data were all generated without antigen retrievals, such as hyaluronidase or chondroitinase treatment, to reduce the static hindrance due to a dense matrix, which could potentially influence the results. Since CO6A3 is believed to be enriched in the chondrocyte territorial, i.e. pericellular matrix, the higher abundance of CO6A3 in the superficial region has been attributed to the higher cellularity of this region. However, our results were based on proteomic analyses of a standardised volume of cartilage matrix, excluding cells, thereby reflecting the absolute abundance in the matrix. We know from our previous study that in situ digestion of cartilage yields $\sim 3$-fold more collagen type VI than guanidine- $\mathrm{HCl}$ extraction, suggesting that a large proportion of collagen type VI may be associated with a guanidine- $\mathrm{HCl}-$ resistant collagen framework (Hsueh et al., 2016). Given that this collagen framework is likely much less accessible to antibody penetration than the proteoglycan component, prior reports of $\mathrm{CO} 6 \mathrm{~A} 3$ localisation seem to have underestimated total CO6A3, instead identifying in the superficial layer a more accessible subpopulation of CO6A3 associated with proteoglycans (Hsueh et al., 2016; Müller et al., 2014).

Many studies have made efforts to understand articular cartilage protein heterogeneity using proteomic methods. Even though the strategies used have been different, the results are consistent. For instance, we observed an enrichment of the aggrecan G3 domain in the interterritorial matrix of OA cartilage compared with an enrichment in the territorial matrix of healthy cartilage. These observations are consistent with previous studies reporting matrix reorganisation. These studies include the existence of different proteoglycan 
pools within the cartilage (Maroudas et al., 1998), the observation of radiolabelled newly synthesised aggrecan in the territorial regions in control bovine cartilage explant cultures (Handley et al., 2002) and the redistribution of the radiolabelled newly synthesised aggrecan into the interterritorial regions, upon exposure to retinoic acid that leads to cartilage degradation (Handley et al., 2002). Our findings that HPLN1 and CHAD were enriched in hip compared to knee cartilage were also consistent with previous studies, which showed more than 2-fold enrichment in hip compared to knee cartilage (Hsueh et al., 2016; Önnerfjord et al., 2012).

Our finding that mimecan was the only protein showing a significant enrichment in knee cartilage agreed with a previous quantitative study showing mimecan to be the most enriched protein in knee cartilage (4-fold enriched compared to hip cartilage) (Önnerfjord et al., 2012). Mimecan, also known as osteoglycin or osteoinductive factor, belongs to the family of small leucine rich proteoglycans (SLRPs), which are abundant in the bone matrix, cartilage cells and connective tissues and are thought to regulate cell proliferation, differentiation and adhesion (Hamajima et al., 2003). Consistent with our observation of enrichment of mimecan in the articular cartilage superficial zone, zone-specific DNA array analysis demonstrated enrichment of mimecan in superficial compared with deep zones of human and bovine articular cartilage (Grogan et al., 2013).

The LCM system we used consisted of a highlyautomated microscope platform and a pulsed-UV laser system. The system generated a laser microbeam able to cut cartilage matrix along any desired path with a focal point of less than $1 \mu \mathrm{m}$ of diameter. This high-resolution laser $(<1 \mu \mathrm{m})$ enabled the collection of ultrapure, selected material from heterogeneous regions of interest for downstream applications. Given the high resolution of the laser, this method can be applied to the isolation of cartilage tissue from multiple species, both large and small, including bovine (Landis et al., 2005), human (Fukui et al., 2008a; Fukui et al., 2008b), rat (Mori et al., 2014) and murine (Landis et al., 2003; Yamane et al., 2007) species. However, none of these studies evaluated the subregional differences. Rather, all these studies focused on genomic profiling and expression differences within cartilage depth. In our present study, we demonstrated the feasibility, even with very limited material $\left(0.02 \pm 0.001 \mathrm{~mm}^{3}\right)$, of evaluating the subregional composition of the extracellular matrix by combining LCM and proteomic methodology. This methodology is very applicable to small animal tissues, since the laser focal point $(<1 \mu \mathrm{m})$ is much thinner than the thickness of their cartilage tissue (e.g. $30 \mu \mathrm{m}$ for mouse distal femoral articular cartilage and $300 \mu \mathrm{m}$ for guinea pig tibial articular cartilage) (Aigner et al., 2010). However, because these small animal articular cartilages are more hypercellular than human tissue, it is to be expected that more time and cost may be involved in collecting sufficient samples for analysis. This methodology could be applied to analyse the therapeutic efficiency of drugs or cartilage repair strategies in OA cartilage animal model systems and thereby, in the long run, advance treatments for human ostearthritis.

This study was limited in its approach due to its focus on candidate proteins, albeit the larger number studied compared to previously published work, and few patient specimens, although all results were adjusted for specimen age. Among the surprising results provided by the proteomic analysis, there was the clear evidence of collagen VI protein expression beyond the territorial matrix. Unfortunately, no robust method, such as a well-validated ELISA, was available to confirm these intriguing collagen VI proteomic results. Expanding this paradigmatic investigation to the whole proteome by non-targeted proteomic approaches will be advantageous in future to gain an even more detailed understanding of cartilage compositional changes due to joint site, disease, depth and subregion.

In this study, we utilised a comprehensive methodology for profiling the composition of cartilage across subregions by combining laser capture microscopy and proteomics. This protocol provided a direct and thorough mean for investigating cartilage overcoming some inherent difficulties in analysing this matrix-rich tissue. Targeting multiple important matrix proteins provided a paradigm for a holistic investigation of cartilage tissue composition. This study revealed that degradation of the interterritorial region of articular cartilage is one of the main consequences of ostearthritis.

\section{Acknowledgments}

This study was supported by OARSI Collaborative Scholarship to M-FH, Collaborative Exchange Award from the Orthopaedic Research Society to VBK and NIH/NIA P30-AG-028716. Mass spectrometers were funded by the Crafoord Foundation and Inga-Britt and Arne Lundberg Foundation and other financial support were obtained from the Swedish Research Council (2010-2889) to PÖ.

The authors have no conflict of interest of any kind with regard to this work.

\section{References}

Abdi H (2010) Holm's sequential Bonferroni procedure. Encyclopedia of research design 1: DOI: http://dx.doi.org/10.4135/9781412961288.n178.

Aigner T, Cook JL, Gerwin N, Glasson SS, Laverty S, Little CB, Mcllwraith W, Kraus VB (2010) Histopathology atlas of animal model systems 
- overview of guiding principles. Osteoarthritis Cartilage 18 Suppl 3: S2-6.

Bhosale AM, Richardson JB (2008) Articular cartilage: structure, injuries and review of management. Br Med Bull 87: 77-95.

Billinghurst RC, Dahlberg L, Ionescu M, Reiner A, Bourne R, Rorabeck C, Mitchell P, Hambor J, Diekmann O, Tschesche H, Chen J, Van Wart H, Poole AR (1997) Enhanced cleavage of type II collagen by collagenases in osteoarthritic articular cartilage. J Clin Invest 99: 1534-1545.

Buckwalter JA, Mankin HJ (1998) Articular cartilage: tissue design and chondrocyte-matrix interactions. Instr Course Lect 47: 477-486.

Buckwalter JA, Mankin HJ, Grodzinsky AJ (2005) Articular cartilage and osteoarthritis. Instr Course Lect 54: 465-480.

Darling EM, Wilusz RE, Bolognesi MP, Zauscher S, Guilak F (2010) Spatial mapping of the biomechanical properties of the pericellular matrix of articular cartilage measured in situ via atomic force microscopy. Biophys J 98: 2848-2856.

DiCesare PE, Morgelin M, Carlson CS, Pasumarti S, Paulsson M (1995) Cartilage oligomeric matrix protein: isolation and characterization from human articular cartilage. J Orthop Res 13: 422-428.

Eyre DR, Weis MA, Wu JJ (2006) Articular cartilage collagen: an irreplaceable framework? Eur Cell Mater 12: 57-63.

Fukui N, Ikeda Y, Ohnuki T, Tanaka N, Hikita A, Mitomi H, Mori T, Juji T, Katsuragawa Y, Yamamoto S, Sawabe M, Yamane S, Suzuki R, Sandell LJ, Ochi $\mathrm{T}$ (2008a) Regional differences in chondrocyte metabolism in osteoarthritis: a detailed analysis by laser capture microdissection. Arthritis Rheum 58: 154-163.

Fukui N, Miyamoto Y, Nakajima M, Ikeda Y, Hikita A, Furukawa H, Mitomi H, Tanaka N, Katsuragawa Y, Yamamoto S, Sawabe M, Juji T, Mori T, Suzuki R, Ikegawa S (2008b) Zonal gene expression of chondrocytes in osteoarthritic cartilage. Arthritis Rheum 58: 3843-3853.

Goldring MB (2012) Articular cartilage degradation in osteoarthritis. HSS Journal 8: 7-9.

Goldring MB, Otero M, Plumb DA, Dragomir C, Favero M, El Hachem K, Hashimoto K, Roach HI, Olivotto E, Borzi RM, Marcu KB (2011) Roles of inflammatory and anabolic cytokines in cartilage metabolism: signals and multiple effectors converge upon Mmp-13 regulation in osteoarthritis. Eur Cell Mater 21: 202-220.

Grogan SP, Duffy SF, Pauli C, Koziol JA, Su AI, D'Lima DD, Lotz MK (2013) Zone-specific gene expression patterns in articular cartilage. Arthritis Rheum 65: 418-428.

Guilak F, Alexopoulos LG, Upton ML, Youn I, Choi JB, Cao L, Setton LA, Haider MA (2006) The pericellular matrix as a transducer of biomechanical and biochemical signals in articular cartilage. Ann N Y Acad Sci 1068: 498-512.
Hamajima S, Hiratsuka K, Kiyama-Kishikawa M, Tagawa T, Kawahara M, Ohta M, Sasahara H, Abiko Y (2003) Effect of low-level laser irradiation on osteoglycin gene expression in osteoblasts. Lasers Med Sci 18: 78-82.

Handley CJ, Winter GM, Ilic MZ, Ross JM, Anthony Poole C, Clem Robinson H (2002) Distribution of newly synthesized aggrecan in explant cultures of bovine cartilage treated with retinoic acid. Matrix Biol 21: 579-592.

Henrikson RC (1997) Histology. Williams and Wilkins, Baltimore 10: 122-124.

Hosseininia S, Weis MA, Rai J, Kim L, Funk S, Dahlberg LE, Eyre DR (2016) Evidence for enhanced collagen type III deposition focally in the territorial matrix of osteoarthritic hip articular cartilage. Osteoarthritis Cartilage 24: 1029-1035.

Howes J-M, Bihan D, Slatter DA, Hamaia SW, Packman LC, Knauper V, Visse R, Farndale RW (2014) The Recognition of Collagen and Triple-helical Toolkit Peptides by MMP-13: sequence specificity for binding and cleavage. J Biol Chem 289: 24091-24101.

Hsueh MF, Khabut A, Kjellström S, Önnerfjord P, Kraus VB (2016) Elucidating the molecular composition of cartilage by proteomics. J Proteome Res 15: 374-388.

Landis WJ, Jacquet R, Hillyer J, Lowder E, Yanke A, Siperko L, Asamura S, Kusuhara H, Enjo M, Chubinskaya S, Potter K, Isogai N (2005) Design and assessment of a tissue-engineered model of human phalanges and a small joint. Orthod Craniofac Res 8: 303-312.

Landis WJ, Jacquet R, Hillyer J, Zhang J (2003) Analysis of osteopontin in mouse growth plate cartilage by application of laser capture microdissection and RT-PCR. Connect Tissue Res 44 Suppl 1: 28-32.

Lee W, Leddy HA, Chen Y, Lee SH, Zelenski NA, McNulty AL, Wu J, Beicker KN, Coles J, Zauscher S, Grandl J, Sachs F, Guilak F, Liedtke WB (2014) Synergy between Piezo1 and Piezo2 channels confers high-strain mechanosensitivity to articular cartilage. Proc Natl Acad Sci USA 111: E5114-5122.

Lorenzo P, Bayliss MT, Heinegård D (1998) A novel cartilage protein (CILP) present in the mid-zone of human articular cartilage increases with age. J Biol Chem 273: 23463-23468.

Lui JC, Chau M, Chen W, Cheung CSF, Hanson J, Rodriguez-Canales J, Nilsson O, Baron J (2015) Spatial regulation of gene expression during growth of articular cartilage in juvenile mice. Pediatr Res 77: 406-415.

Maroudas A, Bayliss MT, Uchitel-Kaushansky N, Schneiderman R, Gilav E (1998) Aggrecan turnover in human articular cartilage: use of aspartic acid racemization as a marker of molecular age. Arch Biochem Biophys 350: 61-71.

Mori Y, Chung UI, Tanaka S, Saito T (2014) Determination of differential gene expression profiles in superficial and deeper zones of mature 
rat articular cartilage using RNA sequencing of laser microdissected tissue specimens. Biomed Res 35: 263 270.

Müller C, Khabut A, Dudhia J, Reinholt FP, Aspberg A, Heinegård D, Önnerfjord P (2014) Quantitative proteomics at different depths in human articular cartilage reveals unique patterns of protein distribution. Matrix Biol 40: 34-45.

Önnerfjord P, Khabut A, Reinholt FP, Svensson O, Heinegård D (2012) Quantitative proteomic analysis of eight cartilaginous tissues reveals characteristic differences as well as similarities between subgroups. J Biol Chem 287: 18913-18924.

Pfister BE, Aydelotte MB, Burkhart W, Kuettner KE, Schmid TM (2001) Del1: a new protein in the superficial layer of articular cartilage. Biochem Biophys Res Commun 286: 268-273.

Priam S, Bougault C, Houard X, Gosset M, Salvat C, Berenbaum F, Jacques C (2013) Identification of soluble 14-3-3 as a novel subchondral bone mediator involved in cartilage degradation in osteoarthritis. Arthritis Rheum 65: 1831-1842.

Rawlings ND, Barrett AJ, Bateman A (2010) MEROPS: the peptidase database. Nucleic Acids Research 38: D227-D233.

Soder S, Hambach L, Lissner R, Kirchner T, Aigner T (2002) Ultrastructural localization of type VI collagen in normal adult and osteoarthritic human articular cartilage. Osteoarthritis Cartilage 10: 464470.

Teeple E, Elsaid KA, Fleming BC, Jay GD, Aslani K, Crisco JJ, Mechrefe AP (2008) Coefficients of friction, lubricin, and cartilage damage in the anterior cruciate ligament-deficient guinea pig knee. J Orthop Res 26: 231-237.

Wang Y, Li Y, Khabut A, Chubinskaya S, Grodzinsky AJ, Önnerfjord P (2016) Quantitative proteomics analysis of cartilage response to mechanical injury and cytokine treatment. Matrix Biol: DOI: 10.1016/j.matbio.2016.12.004.

Wilusz RE, Zauscher S, Guilak F (2013) Micromechanical mapping of early osteoarthritic changes in the pericellular matrix of human articular cartilage. Osteoarthritis Cartilage 21: 1895-1903.

Wu JJ, Weis MA, Kim LS, Eyre DR (2010) Type III collagen, a fibril network modifier in articular cartilage. J Biol Chem 285: 18537-18544.

Yamane S, Cheng E, You Z, Reddi AH (2007) Gene expression profiling of mouse articular and growth plate cartilage. Tissue Eng 13: 2163-2173.

Zhen EY, Brittain IJ, Laska DA, Mitchell PG, Sumer EU, Karsdal MA, Duffin KL (2008) Characterization of metalloprotease cleavage products of human articular cartilage. Arthritis Rheum 58: 2420-2431.

\section{Discussion with Reviewers}

Audrey McAlinden: You have described differences in expression and abundance of collagen type III neo-epitopes. Did you analyse total collagen type II and collagen type II neo-epitopes levels? Differences in expression levels would be expected when comparing, for example, healthy and diseased tissues. Authors: In the target panel we included collagen type II neo-epitope, as well as, its pro-peptides, but no peptides from the triple-helical region, since this region would be cross-linked and, therefore, poorly extracted. However, we did not obtain sufficient signal from these collagen type II peptides and, as a consequence, we cannot draw any general conclusions about this protein.

Editor's Note: The Scientific Editor in charge of this paper was Brian Johnstone. 